\title{
Long title: Attachment and therapeutic alliance in psychological therapy for people with recent onset psychosis who use cannabis
}

\section{Short title: Attachment and therapeutic alliance}

Katherine Berry ${ }^{1 *}$, Tom Palmer ${ }^{2}$, Lynsey Gregg $^{1}$, Christine Barrowclough $^{1} \&$ Fiona Lobban $^{2}$

${ }^{1}$ School of Health Sciences, Division of Psychology and Mental Health, University of Manchester, UK

${ }^{2}$ School of Health and Medicine Division of Health Research, Lancaster University, UK

*Address for correspondence

Division of Psychology and Mental Health, School of Health Sciences, Faculty of Biology, Medicine and Health, University of Manchester, Manchester Academic Health Science Centre, $2^{\text {nd }}$ Floor, Zochonis Building, Brunswick Street, Manchester, M13 9PL, UK.

$\underline{\text { Katherine.Berry@ manchester.ac.uk }}$

\section{Acknowledgements}

We are grateful to staff in the participating NHS Trusts who assisted with the trial, to the research assistants and trial therapists who completed the assessments and to the trial participants.

\section{Financial support}

This article represents research commissioned by the UK's National Institute for Health Research (NIHR) under its Programme Grants for Applied Research scheme (RP-PG-06061302). 


\section{Trial registration details}

ISRCTN: 88275061

HELPER Programme (Substance Misuse) - A phase-specific psychological therapy for people with problematic cannabis use following a first episode of psychosis (ReCAP)

http://www.isrctn.com/ISRCTN88275061

\section{Conflict of interest}

None 


\begin{abstract}
We examine associations between client attachment style and therapeutic alliance in a three arm randomised controlled trial of brief Motivational Interviewing and Cognitive Behavioural Therapy (MICBT) compared with longer term MICBT or standard care alone. Client self-report measures of attachment style were completed at baseline and both clients and therapists in the treatment arms of the trial completed alliance measures one month into therapy. We found that insecure-anxious attachment was positively associated with therapistrated alliance, whereas clients with insecure-avoidant attachment were more likely to report poorer bond with therapist. There was no evidence that client attachment significantly predicted clinical or substance misuse outcomes either directly or indirectly via alliance. Nor evidence that the length of therapy offered interacted with attachment to predict alliance.
\end{abstract}

Key words: alliance; attachment; psychosis; cognitive behavioural therapy; motivational interviewing; substance misuse

\title{
INTRODUCTION
}

A growing body of research has investigated early caregiving relationships and attachment patterns in psychosis (Gumley, Taylor, MacBeth \& Schwannauer, 2014). Attachment theory proposes that children develop internal working models of the self and others through early relationships with caregivers and these working models are carried forward into adulthood (Bowlby, 1969). Infants form secure attachments when their caregiver is typically sensitive and responsive to their needs. In the context of abuse, maltreatment or consistently unresponsive or insensitive care, the individual develops an 
insecure attachment pattern and difficulties in forming relationships with others and regulating affect.

Research has found associations between attachment difficulties and symptoms in psychosis using cross-sectional designs (Gumley, Taylor et al., 2014). One important development in this literature is a prospective study investigating the role of attachment in predicting psychiatric recovery in first episode psychosis (Gumley, Schwannauer, et al., 2014). This study assessed attachment using the Adult Attachment Interview (AAI; George, Kaplan, \& Main, 1985) in a sample of 79 participants and found that greater attachment security, as well as shorter duration of untreated psychosis and greater insight, predicted recovery from negative symptoms at 12 months. Both duration of untreated psychosis and insight also predicted recovery from positive symptoms at 12 months. There was no direct association between attachment security and positive symptoms at 12 months. However, path analysis demonstrated that attachment security was associated with better insight at baseline and shorter duration of untreated psychosis and the relationship between attachment and positive symptoms at 12 months was mediated by baseline insight and duration of untreated psychosis. These findings suggest that attachment security may exert an influence on recovery from positive symptoms by acting on duration of untreated psychosis and insight. Arguably, people with secure attachment might be more able to see the need to seek help for symptoms and more willing to do so.

A number of other previous studies report associations between attachment and poorer engagement with services in people with psychosis (Gumley, Taylor et al., 2014). For example, Berry et al. (2008) found that clients with higher levels of insecure-avoidant attachment, which is characterised by negative beliefs about others, mistrust of others and 
withdrawal from social relationships, were hostile in therapeutic relationships and had poor ratings of therapeutic alliance from both staff and client perspectives. Clients with higher levels of anxious-insecure attachment, which is characterised by negative beliefs about self and sensitivity to rejection from others, were overly dependently in therapeutic relationships, but there were no significant associations between insecure-anxious attachment and therapeutic alliance with mental health workers.

Therapeutic alliance is defined as the degree to which staff and clients agree on the goals and tasks of therapeutic work and the emotional bond between the staff and clients (Bordin, 1974). Therapeutic alliance is an important predictor of outcomes in psychiatric treatment settings, including rates of hospitalisation, symptoms and functioning (Priebe, Richardson, Cooney, Adedeji, \& McCabe, 2011). In a previous study we investigated the role of alliance in predicting outcomes for clients with early psychosis and cannabis misuse participating in a three arm RCT comparing brief Motivational Interviewing and Cognitive Behaviour Therapy (MICBT) with longer term MICBT and standard care alone (Barrowclough et al., 2014). The trial found neither therapy conferred benefit over standard care in terms of reductions in frequency or amount of cannabis use, nor any of the symptom or functioning outcomes assessed. However, poor client-rated alliance was associated with poorer total symptoms as measured by the Positive and Negative Syndrome Scale total scores (PANSS; Kay, Flszbein, \& Opler, 1987) and general functioning as measured by the Global Assessment of Functioning scale (GAF; Hall, 1995) at follow-up (controlling for baseline symptoms), suggesting that alliance may play a role in influencing outcomes. Alliance was also poorer in the brief therapy compared to longer term therapy (Berry, Gregg, Lobban, \& Barrowclough, 2016). 
Given the potentially important role of attachment in both therapeutic alliance and recovery from symptoms, in this paper we investigate whether attachment was associated with alliance in the MICBT trial data and whether attachment impacts on client outcomes either directly or via alliance. Finally, we explore whether length of therapy moderates any associations between attachment and alliance or outcomes, given evidence that people with different types of insecure attachment style may engage better with therapies which match their attachment style (e.g. Bakermans-Kranenburg, Juffer, \& van Ijzendoorn, 1998). Specifically, we hypothesised that people with higher levels of insecure-avoidant attachment would find it easier to engage with briefer interventions, whereas those with higher levels of anxious attachment would find it easier to engage with longer therapy.

\section{METHOD}

\section{Participants}

Participants were those taking part in a three arm pragmatic rater-blind randomised controlled trial of brief MI-CBT (up to 12 sessions) plus standard care compared with longer term MI-CBT (up to 24 sessions) plus standard care and standard care alone (Barrowclough et al., 2014).

\section{Procedure}

Ethical approval for the trial was obtained from the Cumbria and Lancashire Research Ethics Committee (08/H1015/82). Following written informed consent and screening assessments to determine whether inclusion criteria were met, participants completed baseline assessment measures in face-to-face to interviews. Participants were then randomly allocated to one of the three treatment arms of the trial. Outcomes were assessed at baseline, 
4.5 months, 9-months and 18-month follow-up. This paper focuses on 9 month data as this is the primary end point for the trial.

\section{Measures}

\section{Outcomes}

The PANSS (Kay et al., 1987) was used to assess symptoms associated with psychosis at baseline and follow-ups and the GAF (Hall, 1995) was used to measure functioning. All raters were trained and their reliability was assessed and maintained throughout the trial. Substance misuse was assessed using the Time Line Follow Back assessment (TLFB; Sobell \& Sobell, 1992) at baseline and follow-ups, which was shown to have good reliability with hair analysis (Barrowclough et al., 2010). The primary outcome for the trial was number of days abstinent from cannabis in the preceding 30 days.

\section{Attachment}

We measured adult attachment using the Psychosis Attachment Measure (PAM) Berry et al., 2008) at baseline only. The PAM is 16-item self-report questionnaire measure which assesses attachment in terms of the two dimensions of attachment anxiety and attachment avoidance, with higher scores reflecting higher levels of insecure attachment (range 0-3). In common with many other self-report measures of attachment, the subscales on the PAM are continuous measures and are not used to categorise people into attachment subtypes. The PAM has been shown to have good reliability and validity (Gumley, Taylor, et al., 2014). Alphas in the present study were .82 for the anxiety subscale and .72 for the avoidance subscale. 


\section{Alliance}

Therapeutic alliance was assessing by therapist and client versions of the 12-item Working Alliance Inventory (WAI; Tracey \& Kokotovic, 1989) completed approximately one month into therapy [mean number of sessions for therapist WAI $=3.76$ (1.32) and mean number of sessions for client WAI $=4.17(1.47)]$. Items are rated on a scale ranging from 1 (never) to 7 (always), with higher scores reflecting a stronger alliance. An overall index of alliance can be computed across the items in addition to three subscales measuring three key components of alliance: agreement on tasks; agreement on goals and bond

\subsection{Data analysis}

We explored associations between attachment and alliance using Pearson's correlations and results of these univariate analyses were used to inform decisions about which potential mediation models to test. Across all mediation models, the attachment variable was the independent variable, the alliance variable was the mediator and the clinical and substance misuse measures were the dependent variables. Interaction effects between therapy-type and attachment were explored using regression models which included therapy type and attachment interaction as an independent variable and alliance as the dependent variable. Data were analysed in accordance with intention to treat principles, using all available data and adopting the conventional 0.05 alpha level for interpreting findings as statistically significant. No a priori power calculation was carried out for this study we were using all data available in the trial.

\section{RESULTS}

\subsection{Sample characteristics (Table 1)}


A total of 110 participants were randomised with 35 allocated to standard care, 38 were allocated to brief therapy and 37 were allocated to longer-term therapy. Data on the substance misuse outcome were collected for 79 (71.8\%) participants and data on the symptom outcome were collected for $71(64.6 \%)$ participants at 9 months. Alliance data were available for 52 participants. There was no evidence that outcomes improved as a result of either of the therapies, but there was significant variability in individual scores with some patients making improvements and others not. Although no norms are available for the attachment data, mean scores were consistent with those reported in other psychosis research (e.g. Berry et al., 2008).

\subsection{Attachment and alliance}

Insecure-anxious attachment was significantly related to better therapist-rated alliance (meaning therapist felt more engaged with people with insecure-anxious attachment styles). In particular, there were significant associations between insecure-attachment anxiety and therapist perceptions of agreement on therapy tasks and their perceptions of the therapeutic bond. However, there were no significant associations between insecure-anxious attachment and client-rated alliance. Insecure-avoidant attachment was associated with poorer clientrated alliance, but this effect was not statistically significant given the reduced sample size in this analysis. The association between insecure-avoidant attachment and the client's perception of therapeutic bond did, however, reach significance. There were no significant associations between insecure-avoidant attachment and therapist-rated alliance.

\subsection{Attachment and outcome measures}

In the previous paper, we reported that alliance was associated with total symptom and functioning scores (Berry et al., 2016). On the basis of the results of the univariate 
analyses exploring associations between attachment and alliance, we fitted models six mediation models depicted in Figure 1. There was some evidence to suggest that client alliance was a significant mediator in the relationship between avoidant attachment and both PANSS and GAF scores as evidenced by a significant indirect effect in the model. However, this effect was no longer statistically significant when baseline symptoms or functioning scores were controlled for. There was no evidence of statistically significant mediation in any of the other models tested.

\subsection{Interaction between attachment and length of therapy: effect on alliance}

As shown in Tables 4 and 5, contrary to hypotheses there was no evidence that attachment interacted with therapy type to predict client alliance.

\section{DISCUSSION}

We found some evidence the client avoidant attachment was associated with client-rated alliance and the bond component of alliance in particular, suggesting that clients with higher levels of avoidant attachment reported poorer bonds with their therapists. We also found that client anxious attachment was positively associated with therapist-rated alliance, suggesting that therapists reported better therapeutic relationships with clients with high levels of anxious attachment. We found no direct relationship between attachment and client outcomes and the hypothesised moderation and mediation effects were also non-significant when baseline symptoms or functioning were controlled.

The negative association between client attachment avoidance and client-rated alliance is consistent with previous research examining attachment and alliance in people with more established histories of psychosis and their mental health key workers (Berry et al., 2008). 
The finding here that attachment avoidance and attachment anxiety could have different implications for clients and therapists is also potentially important. For example, in the case of clients with avoidant attachment styles, therapists may be unaware of the poorer alliance and as such not highlight issues within supervision or address them in therapy. Similarly, positive associations between therapist alliance and client anxious attachment, but not between client alliance and anxious attachment may suggest that therapists might be vulnerable to assuming clients are well engaged in therapy. Potentially clients with anxious attachment styles who seek closeness in relationships with others due to underlying fears of rejection may try to please therapists which may lead therapists to report positive alliances, despite the fact that clients may be struggling within the therapeutic relationship due to hypersensitivity to signs of rejection from therapists.

As reported in our previous paper, client-alliance was a predictor of symptom and functioning outcomes across both types of therapy (Berry et al., 2016). Given evidence of associations between client attachment and alliance in this data set and previously reported associations between attachment and clinical outcomes including symptoms (Gumley, Taylor, et al., 2014), functioning (Gumley, Taylor, et al., 2014) substance misuse (Allen, Hauser, \& Borman-Spurrell, 1996), we hypothesised that attachment might also predict outcomes either directly or mediated by alliance. However, findings from this relatively small data set suggest that attachment did not impact significantly on outcomes, particularly when baseline scores were controlled. This finding might suggest that it is not important to consider clients' attachment styles in psychological therapy. Nonetheless, we would argue it is too premature to draw such a conclusion due to previously report associations between attachment and clinical outcomes and the fact that the hypothesised mediation models were assessed in a relatively small sample with missing data for alliance and alliance and attachment only captured at one point in time. 
Similarly, we found no evidence for the hypothesised moderation effects of type of therapy on the relationship between attachment and alliance. On the basis of a small body of literature suggesting that clients with different types of attachment might benefit from different types of therapy (Bakermans-Kranenburg et al., 1998; McBride, Atkinson, Quilty, \& Bagby, 2006; Tasca et al., 2006) we hypothesised that that people with higher levels of insecure-avoidant attachment who experience difficulty in forming close relationships with others would find it easier to engage with briefer interventions, whereas those with higher levels of anxious attachment who have greater need for dependency would find it easier to engage with longer therapy. However, the absence of such interaction effects in our dataset does not mean that attachment may not be important to consider in the 'what works for whom' debate. The exploration of the effects of long versus short therapy was an opportunistic analysis as opposed to one that was preconceived at the stage of designing the study. Past research has suggested that factors such as mode of delivery and the type of therapeutic approach may be important factors to consider.

The strengths of this study are the longitudinal design and the inclusion of measures of relational processes such as attachment and alliance. However the study is limited by the relatively small sample size and missing alliance data. An additional problem with our study is that we only report alliance ratings at one-point in time. It is known that therapeutic alliance can change over time (Lecomte, Laferrière-Simard, \& Leclerc, 2012; Lecomte, Leclerc, Wykes, Nicole, \& Baki, 2015). Previous studies measuring alliance over time have suggested that both fluctuations in alliance are themselves predictive of outcomes and that good therapeutic alliances are especially important at specific moments in therapy, namely when more difficult psychological work is done (Lecomte et al., 2012; Lecomte et al., 2015). 
An increasing number of trials of psychological therapy include process measures such as alliance to help researchers understand how the therapy works. We recommend that these studies should assess alliance at multiple points in time and also include measures of attachment style to provide more robust tests of the models presented in this study. We would also argue that such studies should include a measure of attachment at both baseline and end of therapy, given relatively consistent evidence that clients' attachment styles changes as a result of psychological therapy (Taylor, Rietzschel, Danquah, \& Berry, 2014).

\section{References}

Allen, J. P., Hauser, S. T., \& Borman-Spurrell, E. (1996). Attachment theory as a framework for understanding sequelae of severe adolescent psychopathology: An 11-year follow-up study. Journal of Consulting and Clinical Psychology, 64(2), 254-263.

Bakermans-Kranenburg, M. J., Juffer, F., \& van ljzendoorn, M. H. (1998). Interventions with video feedback and attachment discussions: Does type of maternal insecurity make a difference? Infant Mental Health Journal, 19(2), 202-219. doi: Doi 10.1002/(Sici)10970355(199822)19:2<202::Aid-Imhj8>3.0.Co;2-P

Barrowclough, Haddock, G., Wykes, T., Beardmore, R., Conrod, P., Craig, T., ... Tarrier, N., Wykes, T. (2010). Integrated motivational interviewing and cognitive behavioural therapy for people with psychosis and comorbid substance misuse: randomised controlled trial. BMJ: British Medical Journal, 341.

Barrowclough, C., Marshall, M., Gregg, L., Fitzsimmons, M., Tomenson, B., Warburton, J., \& Lobban, F. (2014). A phase-specific psychological therapy for people with problematic cannabis use following a first episode of psychosis: a randomized controlled trial. Psychological Medicine, 44(13), 2749-2761. doi: 10.1017/\$0033291714000208

Berry, Barrowclough, C., \& Wearden, A. (2008). Attachment theory: a framework for understanding symptoms and interpersonal relationships in psychosis. Behaviour Research and Therapy, 46(12), 1275-1282.

Berry, K., Gregg, L., Lobban, F., \& Barrowclough, C. (2016). Therapeutic alliance in psychological therapy for people with recent onset psychosis who use cannabis. Comprehensive Psychiatry, 67, 73-80. doi: 10.1016/j.comppsych.2016.02.014

Bordin, E. S. (1974). Research strategies in psychotherapy. New York,: Wiley.

Bowlby, J. (1969). Attachment. Attachment and Loss Vol. I. London: Hogarth.

George, C., Kaplan, N., \& Main, M. (1985). The Adult Attachment Interview. Unpublished manuscript. Department of Psychology University of California. Los Angeles, Berkeley.

Gumley, A. I., Schwannauer, M., Macbeth, A., Fisher, R., Clark, S., Rattrie, L., . . Birchwood, M. (2014). Insight, duration of untreated psychosis and attachment in first-episode psychosis: prospective study of psychiatric recovery over 12-month follow-up. British Journal of Psychiatry, 205(1), 60-67. doi: DOI 10.1192/bjp.bp.113.126722

Gumley, A. I., Taylor, H. E. F., Schwannauer, M., \& MacBeth, A. (2014). A systematic review of attachment and psychosis: measurement, construct validity and outcomes. Acta Psychiatrica Scandinavica, 129(4), 257-274. doi: 10.1111/acps.12172 
Hall, R. (1995). Global assessment of functioning - a modified scale. Psychosomatics, 36(3), 267-275.

Kay, S. R., Flszbein, A., \& Opler, L. A. (1987). The Positive and Negative Syndrome Scale (PANSS) for Schizophrenia. Schizophrenia Bulletin, 13(2), 261-276.

Lecomte, T., Laferrière-Simard, M.-C., \& Leclerc, C. (2012). What Does the Alliance Predict in Group Interventions for Early Psychosis? [journal article]. Journal of Contemporary Psychotherapy, 42(2), 55-61. doi: 10.1007/s10879-011-9184-2

Lecomte, T., Leclerc, C., Wykes, T., Nicole, L., \& Baki, A. A. (2015). Understanding process in group cognitive behaviour therapy for psychosis. Psychology and Psychotherapy-Theory Research and Practice, 88(2), 163-177. doi: 10.1111/papt.12039

McBride, C., Atkinson, L., Quilty, L. C., \& Bagby, R. M. (2006). Attachment as moderator of treatment outcome in major depression: A randomized control trial of interpersonal psychotherapy versus cognitive behavior therapy. Journal of Consulting and Clinical Psychology, 74(6), 10411054. doi: 10.1037/0022-006X.74.6.1041

Priebe, S., Richardson, M., Cooney, M., Adedeji, O., \& McCabe, R. (2011). Does the therapeutic relationship predict outcomes of psychiatric treatment in patients with psychosis? A systematic review. Psychotherapy and Psychosomatics, 80(2), 70-77. doi: 10.1159/000320976

Sobell, L. C., \& Sobell, M. B. (1992). Timeline follow-back Measuring alcohol consumption (pp. 4172): Springer.

Tasca, G. A., Ritchie, K., Conrad, G., Balfour, L., Gayton, J., Lybanon, V., \& Bissada, H. (2006). Attachment scales predict outcome in a randomized controlled trial of two group therapies for binge eating disorder: An aptitude by treatment interaction. Psychotherapy Research, 16(1), 106-121.

Taylor, P., Rietzschel, J., Danquah, A., \& Berry, K. (2014). Changes in attachment representations during psychological therapy. Psychotherapy Research, 25(2), 222-238. doi: 10.1080/10503307.2014.886791

Tracey, T. J., \& Kokotovic, A. M. (1989). Factor structure of the Working Alliance Inventory. Psychological Assessment, 1(3), 207. 
Table 1: Sample characteristics for trial participants at baseline with WAI data

$$
\mathrm{N}=52
$$

Age in years, mean (SD)

24

(4.96)

Gender: Male, n (\%)

47

$(90.4 \%)$

History of psychosis in months, mean (SD)

17

$(13.56)$

History of cannabis use in years, mean (SD)

10

PANSS total, mean (SD),

62.92

(13.19)

Global functioning (GAF), mean (SD)

36.92

Number of days abstinent from cannabis in preceding 30 day period, mean (SD)

Client alliance (WAI), mean (SD),

Therapist alliance (WAI), mean (SD),

Attachment avoidance (PAM), mean (SD),

Attachment anxiety (PAM), mean (SD),

$\begin{array}{cl}12.58 & (10.70) \\ & \\ 61.97 & (9.25) \\ 60.32 & (9.01) \\ 1.76 & (.57) \\ 1.18 & (.66)\end{array}$


Table 2: Correlation matrix for attachment and alliance variables

\section{PAM avoidance PAM Anxiety}

\begin{tabular}{|c|c|c|}
\hline WAI client & $r=-.305$ & $r-=-.064$ \\
\hline Total & $\mathrm{p}=.075$ & $\mathrm{p}=.715$ \\
\hline WAI client & $\mathrm{r}=-.203$ & $\mathrm{r}=-.043$ \\
\hline Tasks & $\mathrm{p}=.242$ & $p=.808$ \\
\hline WAI client & $\mathrm{r}=-.298$ & $\mathrm{r}=-.082$ \\
\hline Goal & $\mathrm{p}=.082$ & $p=.641$ \\
\hline WAI client & $r=-.334 *$ & $r=-.064$ \\
\hline Bond & $\mathrm{p}=.05$ & $\mathrm{p}=.715$ \\
\hline WAI therapist & $r=-.100$ & $r=.284^{*}$ \\
\hline Total & $\mathrm{p}=.480$ & $\mathrm{p}=.041$ \\
\hline
\end{tabular}




\begin{tabular}{lcc}
\hline WAI therapist & $\mathrm{r}=-.080$ & $\mathrm{r}=.329^{*}$ \\
Tasks & $\mathrm{p}=.574$ & $\mathrm{p}=.017$ \\
& $\mathrm{r}=-.052$ & $\mathrm{r}=.196$ \\
WAI therapist & $\mathrm{p}=.174$ & \\
Goals & $\mathrm{p}=.164$ \\
& $\mathrm{r}=-.166$ & $\mathrm{r}=.280^{*}$ \\
WAI therapist & $\mathrm{p}=.238$ & $\mathrm{p}=.045$ \\
Bond &
\end{tabular}




\begin{tabular}{|c|c|c|c|c|c|}
\hline PAM anxiety & $\begin{array}{l}\text { Days } \\
\text { abstinence }\end{array}$ & WAI-T & $\begin{array}{l}.1 .45 \\
(-.02-4.10)\end{array}$ & $\begin{array}{l}2.36 \\
(-3.42-8.14)\end{array}$ & $\begin{array}{l}3.81 \\
(-1.74-9.37)\end{array}$ \\
\hline
\end{tabular}

Table 4: Model summary for interaction between insecure-anxious attachment and therapy in predicting in predicting client alliance

\begin{tabular}{llll}
\hline Variable & Beta & $\mathrm{p}$ & $\begin{array}{l}95 \% \text { confidence } \\
\text { intervals }\end{array}$ \\
\hline Therapy type & .41 & .215 & $-4.56-19.47$ \\
PAM anxiety & -.19 & .387 & $-8.84-3.52$ \\
Therapy type X & .13 & .725 & $-7.37-10.47$ \\
PAM anxiety & & & \\
\hline$\left(\mathrm{R}^{2}\right.$ complete model $\left.=.26, \mathrm{p}=.026\right)$ & &
\end{tabular}

Table 5: Model summary for interaction between insecure-anxious attachment and therapy type in predicting client alliance

\begin{tabular}{llll}
\hline Variable & Beta & $\mathrm{P}$ & $\begin{array}{l}95 \% \text { confidence } \\
\text { intervals }\end{array}$ \\
\hline Therapy type & .44 & .013 & $1.79-14.27$ \\
PAM avoidance & -.16 & .314 & $-8.69-3.10$ \\
Therapy type X & -.10 & .774 & $-13.47-10.12$ \\
PAM avoidance & & \\
\hline (R complete model $=.26, \mathrm{p}=.025)$ &
\end{tabular}


Figure 1: Diagrammatic representation of mediation models

\begin{tabular}{|l|l|l|l|}
\hline $\begin{array}{l}\text { Client avoidance } \\
\text { attachment }\end{array}$ & Client alliance & $\begin{array}{l}\text { Symptoms } \\
\text { Functioning } \\
\text { Substance use }\end{array}$ \\
\cline { 2 - 3 } & $\longrightarrow$ \\
\end{tabular}

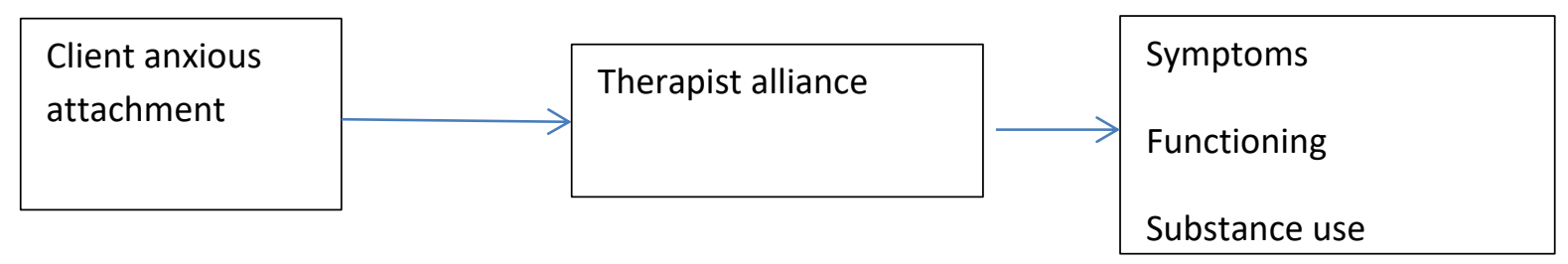

